\title{
Transverse Movement of the Median Nerve in the Carpal Tunnel during Wrist and Finger Motion in Patients with Carpal Tunnel Syndrome
}

\author{
Mitsuhiko Nanno, ${ }^{1}$ Takuya Sawaizumi, ${ }^{1}$ Norie Kodera, ${ }^{1}$ Yuji Tomori ${ }^{1}$ and \\ Shinro Takai ${ }^{1}$
}

${ }^{1}$ Department of Orthopaedic Surgery, Nippon Medical School, Tokyo, Japan

\begin{abstract}
Carpal tunnel syndrome (CTS) is the most common peripheral compression neuropathy of the upper extremity. Repetitive wrist and finger motion has been suggested as a major factor of pathogenesis of CTS. However, little is known about the pathomechanics of CTS. We aimed to evaluate the movement of the median nerve in the carpal tunnel during wrist and finger motions using transverse ultrasound in 21 patients with CTS (5 men and 16 women with mean age 69.0 years). We examined quantitatively the median nerve location as a coordinate within the carpal tunnel at varied wrist positions with all fingers full extension and flexion respectively in the affected and unaffected sides. We thus found that at all wrist positions during finger motion, the median nerve moved significantly more ulnopalmarly in the affected side compared to the unaffected side $(p<0.05)$. Especially, at the wrist palmar-flexion position as a provocative test, the nerve moved significantly $(p<0.05)$ the most ulnopalmarly among all wrist positions in the affected side. The nerve was the most strongly compressed against the transverse carpal ligament by the flexor tendons. Additionally, the displacement amount of the nerve in the dorsal-palmar direction was significantly smaller in the affected side than in the unaffected side. These findings indicate that such a pattern of nerve movement has the potential to distinguish affected from unaffected individuals. This ultrasound information could be useful in better understanding of the pathomechanics of CTS, and in further improvement of diagnosis and treatment for CTS.
\end{abstract}

Keywords: carpal tunnel syndrome; finger motion; median nerve; ultrasound; wrist position Tohoku J. Exp. Med., 2015 July, 236 (3), 233-240. 다이 2015 Tohoku University Medical Press

\section{Introduction}

It is generally accepted that repetitive wrist and finger motion is a risk factor for carpal tunnel syndrome (CTS) (Zhao et al. 2007). Moreover, the median nerve and the tendon movement during such repetitive motion may contribute to CTS (Ugbolue et al. 2005). In addition, these repetitive tasks may cause pathological changes in the nerve and the tendons (Ugbolue et al. 2005). However, to date, its exact pathogenesis remains unknown (Ugbolue et al. 2005; Yoshii et al. 2009).

In recent years, several studies have demonstrated that wrist and finger motion could bring substantial movement of the median nerve and the flexor tendons at the wrist (Yoshii et al. 2009, 2013; van Doesburg et al. 2010, 2012; Wang et al. 2014a, b; Nanno et al. 2015). Additionally, the transverse displacements of the nerve and the tendons within the carpal tunnel have been evaluated during wrist or finger motion (Nakamichi and Tachibana 1992; Erel et al.
2003). Some studies by transverse ultrasound have shown that the median nerve moves palmarly in active finger flexion, and gets compressed between the transverse carpal ligament (TCL) and the flexor tendons in healthy subjects and in patients with CTS (Yoshii et al. 2009; van Doesburg et al. 2012; Wang et al. 2014a). In addition, the value of the ultrasound examinations has been demonstrated for the assessment of both median nerve and carpal tunnel pathology (Vögelin et al. 2014). Evaluating transverse displacement of the median nerve during wrist and finger motion quantitatively may offer useful information about kinematic and pathophysiological changes of the median nerve in CTS patients (Wang et al. 2014a).

Although such wrist and finger motion is important in the pathomechanism of CTS, there has been little research comparing the transverse ultrasound displacement of the median nerve within the carpal tunnel at varied wrist motions (Wang et al. 2014a, b) during finger motion in patients with CTS between the affected side and the unaf-

Received March 30, 2015; revised and accepted June 4, 2015. Published online July 2, 2015; doi: 10.1620/tjem.236.233.

Correspondence: Mitsuhiko Nanno, Department of Orthopaedic Surgery, Nippon Medical School, 1-1-5 Sendagi, Bunkyo-ku, Tokyo 113-8602, Japan.

e-mail: nanno-mi@nms.ac.jp 
fected side. These nerve motion patterns may be useful not only in distinguishing affected from unaffected individuals, but also in studies of the pathogenesis of CTS. Comparison between the affected and unaffected sides of the CTS patients could reduce the problem of individual differences. Furthermore, Yoshii et al. (2013) have described the detailed location of the median nerve as a coordinate within the carpal tunnel. It is important to describe the position of the median nerve as a coordinate to further understand the accurate nerve location within the carpal tunnel during wrist and finger motions.

The objective of this study was to examine quantitatively the displacement of the median nerve, by assigning a coordinate position to the median nerve within the carpal tunnel during wrist and finger motions by transverse ultrasound in the affected side and the unaffected side of CTS patients. Such information can offer further evaluation of the pathomechanics of CTS, and provide a more accurate diagnostic assessment of and better treatment for CTS by such as distinguishing affected from unaffected individuals.

\section{Materials and Methods}

We recruited 21 patients with idiopathic CTS (5 men and 16 women; and mean age 69.0 years with a range of 44-93 years) who were clinically diagnosed and confirmed by electrophysiological studies (mean distal motor latency $8.8 \mathrm{~ms}$ with a range of $5.54-12.45 \mathrm{~ms}$ included 7 patients with electrical silence). CTS patients were excluded if they reported a history of systemic disease associated with a higher incidence of carpal tunnel syndrome, such as rheumatoid arthritis, thyroid disease, or any trauma or surgery of the hand, wrist, or forearm. And the patients with CTS whose wrists were affected bilaterally were also excluded. All patients had symptoms only on one side. In addition, the electrophysiological studies of the hand in the unaffected side were negative for CTS. This study protocol was approved by our institutional review board. After signed consent was obtained from all participants in this study, ultrasound examination of both wrists of the affected side and the unaffected side were proceeded.

The image acquisition procedure has been described previously. In brief, each subject was seated with the elbow in approximately $45^{\circ}$ flexion, the forearm in supination, and the shoulder in neutral position. The forearm of the participant was fixed to a custom-made device with the wrist in the neutral position. We used an ultrasound scanner (Mylab Five, Hitachi Medical Corporation, Tokyo, Japan) equipped with an LA332 3.5/12 MHz Hi-Definition linear array transducer (LA332).

A single orthopedic surgeon performed ultrasound examination in a standardized manner. The transducer was placed at the level of the carpal tunnel with the wrist in the neutral position (Fig. 1). A custom-made device fixed transducer was strapped at the participant's palm to maintain the transducer stable during examination. Additionally, the transducer was held in a $90^{\circ}$ angle to the wrist to iden-

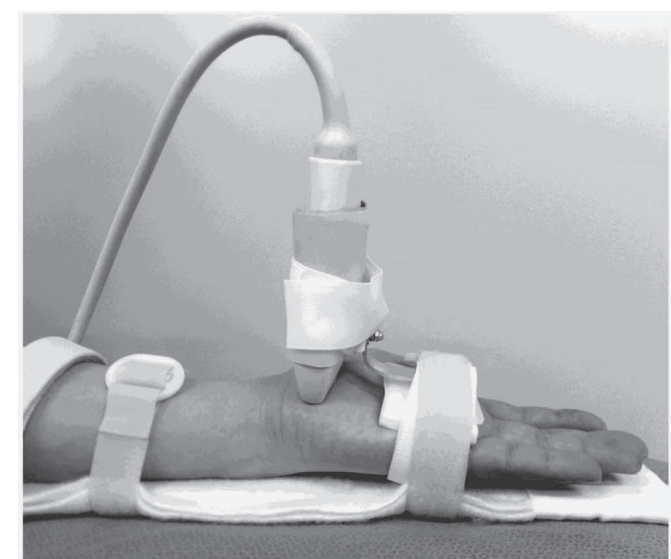

Fig. 1. Setup of transverse ultrasound examination equipment.

tify both the ridge of the trapezium and the hook of the hamate with reference to a protractor attached to the table, without adding extra pressure to avoid compression to the carpal tunnel contents. It is easy to identify these two bony landmarks in all hands. The median nerve, the flexor pollicis longus (FPL), and the flexor digitorum superficialis (FDS) and profundus tendons (FDP) were recognized by transverse ultrasonographic imaging during full flexion and full extension of all five fingers. After acquiring a plain image, the transducer was set in a custom made fixture at each of its five study positions (neutral, $60^{\circ}$ dorsal flexion, $60^{\circ}$ palmar flexion, $40^{\circ}$ ulnar deviation, $10^{\circ}$ radial deviation) during finger motion, respectively. We measured and corrected Each wrist angle using a goniometer (SD1-01, Suzukiiryo, Inc., Tokyo) placed on the back of the hand at each of the five wrist positions. We evaluated both left and right wrists of all participants. The participants moved from full extension to maximum flexion of all five fingers as a fist motion at each wrist position continuously and repeatedly. Two cycles of the flexion-extension finger motion were recorded for each wrist position.

We evaluated all recorded images and selected the initial and final frames of the motion cycle for each of the finger extension and flexion positions at each wrist position. The median nerve and the flexor tendons were identified in both the extension and the flexion positions of the fingers using these images.

We determined the coordinates of the median nerve as follows (Fig. 2). The reference point (P) was defined as the midpoint of the distance (A) between the apex of the ridge of the trapezium and the apex of the hook of the hamate. The distances between the reference point and the centroid of the median nerve on the $\mathrm{X}$-axis $(\mathrm{X})$ and on the $\mathrm{Y}$-axis $(\mathrm{Y})$ were evaluated. The position of the median nerve was defined as a coordinate point (X mm, Y mm). Correcting for individual differences, the ratio $(\mathrm{x})$ of distance $(\mathrm{X})$ to distance (A) and the ratio (y) of distance (Y) to distance (A) were evaluated and defined as the radial deviation ratio and the dorsal deviation ratio of the median nerve respectively. 


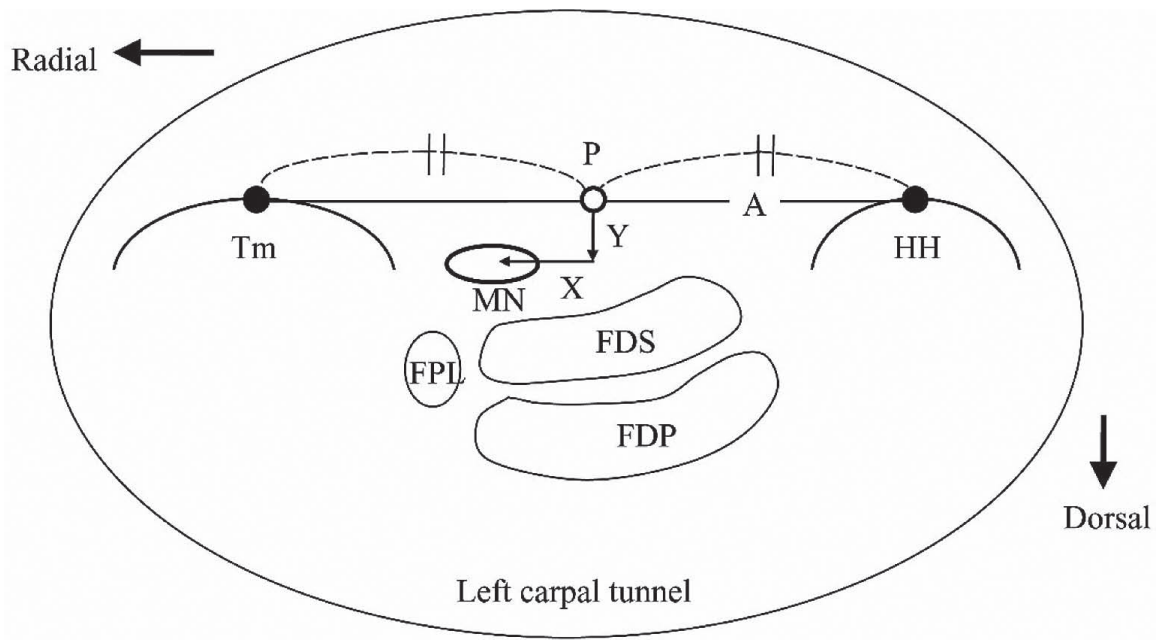

Fig. 2. Illustration of measuring the location of the median nerve within the carpal tunnel as a coordinate.

Distance 'A': the distance between the vertex of the ridge of the Tm and the vertex of the HH. Point ' $\mathrm{P}$ ': the reference point defined as the midpoint of the distance 'A'. Distance ' $\mathrm{X}$ ': The distance between the point ' $\mathrm{P}$ ' and the centroid of $\mathrm{MN}$ on the $\mathrm{X}$-axis. Distance ' $\mathrm{Y}$ ': The distance between the point ' $\mathrm{P}$ ' and the centroid of $\mathrm{MN}$ on the Y-axis. MN, the median nerve; Tm, the trapezium; HH, the hook of the hamate; FPL, the flexor pollicis longus; FDS, the flexor digitorum superficialis; FDP, the flexor digitorum profundus.

The corrected position of the median nerve was demonstrated as a coordinate point $(\mathrm{x}, \mathrm{y})$. The radial and dorsal directions were defined as positive and the ulnar and palmar directions as negative. Hence, the displacement of the nerve in the radial-ulnar and the dorsal-palmar directions could be calculated by comparing with the coordinates at the initial position of the nerve and its final one within the carpal tunnel during finger motion.

All results were mentioned in mean $\pm \mathrm{SD}$. The averages of 3 cycles were calculated for each parameter and used for further analysis. We performed all analyses by SPSS 21.0J. A paired t-test was used to analyze the differences of the median nerve from the neutral wrist position to the other four wrist positions in all five fingers extension in both the affected side and the unaffected side. Furthermore, median nerve displacement amount from full finger extension to full flexion at each of the five wrist positions was statistically compared between the affected side and the unaffected side with non-paired t-test. P-values of $<0.05$ were considered statistical significance for finger and wrist motion differences.

\section{Results}

We have examined the locations of the median nerve in finger extension both in the radial-ulnar and the dorsalpalmar directions at varied wrist positions (Tables 1 and 2). The average of the median nerve at the wrist neutral position in the radial-ulnar direction was $3.54 \pm 0.51$ in the affected side, and $7.89 \pm 0.84$ in the unaffected side (Table 1). The average location of the median nerve at the wrist neutral position in the dorsal-palmar direction was $6.43 \pm$ 1.37 in the affected side and $9.09 \pm 0.92$ in the unaffected side (Table 2).

In the radial-ulnar direction, the median nerve was sit- uated statistically significantly ( $<0.05$ ) more ulnarly both at the wrist palmar and radial deviation positions than at the wrist neutral position in full finger extension in both the affected side and the unaffected side (Table 1). The median nerve displacement to the ulnar side was statistically significantly larger $(\mathrm{p}<0.05)$ at the wrist palmar flexion position than at the radial deviation position. On the other hand, the median nerve was situated statistically significantly $(\mathrm{p}<$ $0.05)$ more radially at the wrist ulnar deviation position than at the wrist neutral position in full finger extension in both the affected side and the unaffected side. The median nerve displacement toward the radial side was statistically significantly larger $(p<0.05)$ at the wrist ulnar deviation position than at the dorsal flexion position. In the dorsalpalmar direction, the median nerve was situated statistically significantly $(\mathrm{p}<0.05)$ more dorsally at the wrist dorsal flexion than it was at the wrist neutral position in full finger extension in both the affected side and the unaffected side (Table 2). Conversely, the median nerve was situated statistically significantly $(p<0.05)$ more palmarly at the wrist palmar, ulnar and radial deviation positions than at the wrist neutral position in full finger extension in both the affected side and the unaffected side.

We also described he median nerve displacement at varied wrist positions during all fingers motion. In the radial-ulnar direction, at the wrist neutral, dorsal, and ulnar deviation positions, the median nerve moved statistically significantly $(p<0.05)$ more radially in full finger flexion than in full finger extension in both the affected side and the unaffected side (Table 1). Conversely, at the wrist palmar and radial deviation positions, the median nerve moved statistically significantly $(\mathrm{p}<0.05)$ more ulnarly in full finger flexion than in full finger extension in both the affected side and the unaffected side. In the dorsal-palmar direction, at 
Table 1. Radial deviation ratio of the median nerve at varied wrist position with finger motion in both the affected and unaffected sides.

\begin{tabular}{|c|c|c|c|c|c|c|c|c|}
\hline \multirow{2}{*}{ Wrisit position } & \multicolumn{4}{|c|}{ Fingers extension } & \multicolumn{4}{|c|}{ Fingers flexion } \\
\hline & Affected side (SD) & & ffected side (SD) & & Affected side (SD & & Jnaffected side (S & \\
\hline Neutral & $3.54(0.51)-$ & * & $7.89(0.84)$ & & $4.81(0.64)$ & $*$ & $9.75(0.84) \sqsupset$ & \\
\hline Dorsal Flexion & $4.77(1.04)$ & $*$ & $8.56(0.68)-$ & & $7.02(1.56)-$ & $*$ & $10.37(1.34)-$ & \\
\hline Palmar Flexion & $-7.66(2.47)-$ & $* *$ & $-1.54(0.85)-$ & $*$ & $-10.61(2.7)$ & $*$ * & $-6.98(1.76)-$ & $*$ \\
\hline Ulnar deviation & $6.34(1.69)-$ & $*$ & $9.32(0.79)-$ & & $8.99(2.53)-$ & $*$ & $12.2(1.71)$ & \\
\hline Radial deviation & $-2.11(1.37)$ & $*$ & $-0.65(0.48)-$ & & $-3.09(0.92)-$ & $*$ & $-2.19(0.96)-$ & \\
\hline
\end{tabular}

$(* \mathrm{p}<0.05)$

Table 2. Dorsal deviation ratio of the median nerve at varied wrist position with finger motion in both the affected and unaffected sides.

\begin{tabular}{|c|c|c|c|c|c|c|c|c|}
\hline \multirow{2}{*}{ Wrisit position } & \multicolumn{4}{|c|}{ Fingers extension } & \multicolumn{4}{|c|}{ Fingers flexion } \\
\hline & Affected side (SD) & \multicolumn{3}{|c|}{ Unaffected side (SD) } & \multicolumn{2}{|l|}{ Affected side (SD) } & \multicolumn{2}{|l|}{ Unaffected side (SD) } \\
\hline Neutral & $6.43(1.37) \neg-$ & * & $9.09(0.92) \sqsupset$ & & $5.42(0.86) \neg^{-}$ & $*$ & $7.53(0.68) \sqsupset$ & \\
\hline Dorsal Flexion & $7.8(1.06)-$ & $*$ & $10.42(1.42)$ & & $6.46(4.61)--$ & * & $8.54(1.72)-$ & \\
\hline Palmar Flexion & $1.56(1.44)-*$ & $* *$ & $6.54(1.18)-$ & * & $0.52(1.32)-$ & $*$ * & $4.8(2.01)-$ & * \\
\hline Ulnar deviation & $5.2(0.96)-$ & * & $8.26(1.1)-$ & & $4.57(3.95)--$ & $*$ & $7.03(0.9)-$ & \\
\hline Radial deviation & $2.31(1.18)$ & $*$ & $7.73(1.43)$ & & $0.97(1.27)$ & $*$ & $5.95(1.33)-$ & \\
\hline
\end{tabular}

$(* \mathrm{p}<0.05)$

all wrist positions, the median nerve moved statistically significantly $(\mathrm{p}<0.05)$ more palmarly in full finger flexion than in full extension in both the affected side and the unaffected side (Table 2). In particular, the median nerve moved statistically significantly $(\mathrm{p}<0.05)$ the most palmarly at the wrist palmar flexion position and the most dorsally at the wrist dorsal flexion position among all wrist positions during finger motion in both the affected side and the unaffected side.

Moreover, we researched the median nerve displacement compared between the affected side and the unaffected side. In the radial-ulnar direction, at all wrist positions in both full finger extension and flexion, the median nerve moved statistically significantly $(\mathrm{p}<0.05)$ more ulnarly in the affected side than in the unaffected side (Table 1). And the median nerve moved statistically significantly $(\mathrm{p}<0.05)$ the most ulnarly at the wrist palmar flexion position of finger flexion in the affected side among all wrist positions during finger motion. In the dorsal-palmar direction, at all wrist positions in both full finger extension and flexion, the median nerve moved statistically significantly $(p<0.05)$ more palmarly in the affected side than in the unaffected side (Table 2). Therefore, the distance between the nerve and the TCL in the dorsal-palmar direction was significantly smaller in the affected side than in the unaffected side $(\mathrm{p}<0.05)$. And the median nerve moved statistically significantly $(\mathrm{p}<0.05)$ the most palmarly at the wrist palmar flexion position of finger flexion in the affected side among all wrist positions during finger motion.
Furthermore, we examined the median nerve displacement amount during finger motion compared between the affected side and the unaffected side. In the radial-ulnar direction, the displacement amount of the median nerve was statistically significantly smaller $(\mathrm{p}<0.05)$ radially at the wrist neutral position, and ulnarly at the wrist palmar and radial deviation positions in the affected side, compared with the unaffected side (Table 3). Especially, it was statistically significantly smallest $(\mathrm{p}<0.05)$ at the wrist palmar flexion position among all wrist positions during finger motion in the affected side, compared with the unaffected side. At the wrist dorsal and ulnar deviation positions, its displacement amount was relatively smaller in the affected side than in the unaffected side, but it did not reach significance.

In the dorsal-palmar direction, at all wrist positions, the displacement amount of the median nerve was statistically significantly smaller $(p<0.05)$ dorsally in the affected side, compared with the unaffected side (Table 3).

\section{Discussion}

Repetitive wrist and finger motion has been suggested as a major factor of pathogenesis of CTS (Ugbolue et al. 2005). However, little is known about the mechanisms by which these motions might cause CTS (Yoshii et al. 2009). In addition, the most commonly reported pathological finding of CTS is noninflammatory fibrosis and thickening of the subsynovial connective tissue (SSCT), which mediates movement between the nerve and tendons (Lluch 1992; Ettema et al. 2004). One of the explanations to associate 
Table 3. Displacement amount of the median nerve from finger extension to finger flexion compared between the affected side and the unaffected side.

\begin{tabular}{|c|c|c|c|c|c|c|c|}
\hline \multirow{2}{*}{ Wrisit position } & \multicolumn{3}{|c|}{ Radial deviation ratio } & & \multicolumn{3}{|c|}{ Dorsal deviation ratio } \\
\hline & Affected side (SD) & & ffected side (SD) & & Affected side (SD) & & affected side (SD) \\
\hline Neutral & $1.27(0.18)$ & $*$ & $1.86(0.18) \neg$ & & $-1.01(0.19)$ & * & $-1.56(0.2)$ \\
\hline Dorsal Flexion & $2.25(2.3)$ & & $2.3(0.19)-$ & & $-1.34(0.58)$ & $*$ & $-1.88(0.3)$ \\
\hline Palmar Flexion & $-2.95(0.85)-$ & $* *$ & $-5.45(2.19)-$ & * & $-1.04(0.8)$ & $*$ & $-1.74(0.54)$ \\
\hline Ulnar deviation & $2.65(0.73)-$ & & $2.87(0.32)-$ & & $-0.63(0.33)$ & * & $-1.23(0.4)$ \\
\hline Radial deviation & $-0.98(0.46)-$ & $*$ & $-1.54(0.91)-$ & & $-1.35(0.23)$ & * & $-1.78(0.25)$ \\
\hline
\end{tabular}

$(* \mathrm{p}<0.05)$

the wrist and finger motion with pathological changes in CTS is thought to be that these motions cause a shearing injury to the SSCT.

Recently, several studies reported that the median nerve moves longitudinally and transversely in response to active tendon motion in the carpal tunnel during finger or wrist movement (Nakamichi and Tachibana 1992; Erel et al. 2003; Yoshii et al. 2009; van Doesburg et al. 2010). Additionally, some authors have used transverse ultrasound to evaluate median nerve motion in the carpal tunnel (Yoshii et al. 2009, 2013; van Doesburg et al. 2010, 2012; Wang et al. 2014a, b; Nanno et al. 2015). With use of this modality, it has previously been determined that the median nerve becomes displaced palmarly and gets compressed against the TCL in active finger flexion in healthy subjects and in patients with CTS (Yoshii et al. 2009, 2013; van Doesburg et al. 2012; Wang et al. 2014a, b).

There are a number of studies concerned with transverse displacement patterns of median nerve in the carpal tunnel during finger motion at the wrist neutral position (Yoshii et al. 2009, 2013; van Doesburg et al. 2010, 2012; Wang et al. 2014a). However, there has been little research detailing the transverse displacement of the median nerve within the carpal tunnel at various wrist positions during finger motion by ultrasound. We have recently described the ultrasound technique to evaluate quantitatively the median nerve movement as a coordinate within the carpal tunnel during wrist and finger motion in limited normal subjects (Nanno et al. 2015). In the present study, using this technique, we evaluated the median nerve displacement within the carpal tunnel during wrist and finger motion in CTS patients. Consequently, at the wrist palmar-flexion position in the affected side, the median nerve moved significantly the most ulnopalmarly among all wrist positions. The motion of wrist palmar flexion is also used commonly as a provocative test, so-called the Phalen test (Tetro et al. 1998), to diagnose CTS. From this finding, it is speculated that the median nerve may be pushed to the ulnopalmar side, and be compressed against the transverse carpal ligament by the flexor tendons the most strongly at wrist palmar position. Moreover, at this wrist position in finger flexion, the nerve moved statistically significantly $(\mathrm{p}<0.05)$ more ulnopalmarly than in finger extension. This finding suggested that symptoms of CTS would be provoked at the wrist palmar flexion position with fist-making finger motion, which might be called the "dynamic Phalen test", than at the wrist palmar position without any finger motion.

Conversely, we observed that the median nerve at the wrist dorsal flexion position was located the most radiodorsally within the carpal tunnel among all wrist positions during finger motion. In addition, the median nerve moved radiopalmarly to escape the compression and additionally shifts away from the transverse carpal ligament in full finger flexion. This finding suggests that the compression or the shearing stress of the median nerve caused by the flexor tendon motion could be the smallest in the wrist dorsal flexion position among all wrist positions. From this study, such a wrist position may be the effective position for a wrist splint in the treatment for CTS.

Recently, some studies on nerve displacement comparing it in healthy subjects and patients with CTS by ultrasound have been reported (Yoshii et al. 2009, 2013; van Doesburg et al. 2010, 2012; Wang et al. 2014a, b). Moreover, some reports described that the transverse ultrasound displacement of the median nerve moved more palmarly or ulnopalmarly in healthy subjects than in CTS patients, similar to our findings (van Doesburg et al. 2012; Yoshii et al. 2013; Wang et al. 2014a). However, no description of the median nerve orientation within the carpal tunnel, such as the start and end points of the nerve during wrist and finger motions was provided, although they evaluated the directions or vectors of the median nerve during finger motion by ultrasound (Yoshii et al. 2009, 2013; van Doesburg et al. 2010, 2012). In addition, there has been little information demonstrating the detailed location of the median nerve as a coordinate in the carpal tunnel (Yoshii et al. 2009, 2013; van Doesburg et al. 2010, 2012). Furthermore, they have only examined its displacement amount as an actual measurement, and provided no correction for individual differences in the carpal tunnel size (Yoshii et al. 2009, 2013; van Doesburg et al. 2010, 2012; Wang et al. 2014a, b). Therefore, it would be difficult to simply compare its displacement amount between the data for healthy subjects and that for CTS patients. In the current study, all locations of the median nerve using the corrected coordinates have been evaluated, and all displace- 
ment amounts have been calculated.

In contrast to studies on the transverse movement of the median nerve compared between healthy subjects and CTS patients (van Doesburg et al. 2012; Yoshii et al. 2013; Wang et al. 2014b), few studies have yet addressed it compared between the affected side and the unaffected side of the same individual in CTS patients. The current study demonstrated a significant difference in the median nerve displacement during wrist and finger motion when comparing that in the affected side and in the unaffected side of the patients with CTS. Consequently, at all wrist positions during finger motion, the median nerve moved statistically significantly $(\mathrm{p}<0.05)$ more ulnopalmarly in the affected side than in the unaffected side (Tables 1 and 2). Nakamichi and Tachibana (1995) reported that the median nerve was adhered to the TCL in patients with CTS from intraoperative observation. Similar observations of fibrosis in CTS patients have been found around the SSCT, which loosely connects the flexor tendons and serves to reduce friction (Ettema et al. 2007). From this finding, it is speculated that there may be adhesion among the nerve, the tendons and the TCL due to fibrosis of the SSCT in the affected side, which could shift the nerve to the TCL, and reduce nerve displacement. In this presence of nerve adhesion, the wrist and finger motion might cause compression and deformation of the nerve, and might develop to CTS (Ettema et al. 2007).

Additionally, we evaluated quantitatively the displacement amount of the median nerve during wrist and finger motion, and compared it from full finger extension to full flexion at varied wrist positions between the affected side and the unaffected side (Table 3). In consequence, at all wrist positions, the displacement amount of the median nerve was smaller, especially in the dorsal-palmar direction, statistically significantly $(\mathrm{p}<0.05)$ in the affected side, compared with the unaffected side. The strength of this study is that the displacement and direction of the median nerve could be quantitatively examined using coordinates. Therefore, we could compare objectively its displacement amount during wrist and finger motion between the affected and the unaffected side. Especially, at the wrist palmar flexion position, the median nerve displacement amount was smallest statistically significantly $(\mathrm{p}<0.05)$ among all wrist positions, compared between the affected side and the unaffected side. Nakamichi and Tachibana (1995) reported decreased transverse displacement of the median nerve in CTS patients compared with healthy subjects, which is in line with our study findings. They also related that this decrease in nerve mobility may be of significance in the pathophysiology of CTS, and that the more the nerve is restricted from moving, the more mechanical stress the nerve is likely to suffer. Liong et al. (2014) described that CTS patients displayed significantly reduced nerve movement relative to healthy subjects by ultrasound. Yoshii et al. (2009) suggested that the nerve was unable to readjust itself and, consequently is subject to localized compression, by which vast repeated wrist and finger motions may result in nerve damage. Our study indicates that CTS patients have decreased nerve displacement in the affected side relative to that in the unaffected side. From these findings, it is speculated that adhesion around the nerve may be the reason for the smaller nerve displacement in the affected side. Additionally, we believe that that the adhesion around the nerve would reduce the mobility of the nerve, and the nerve

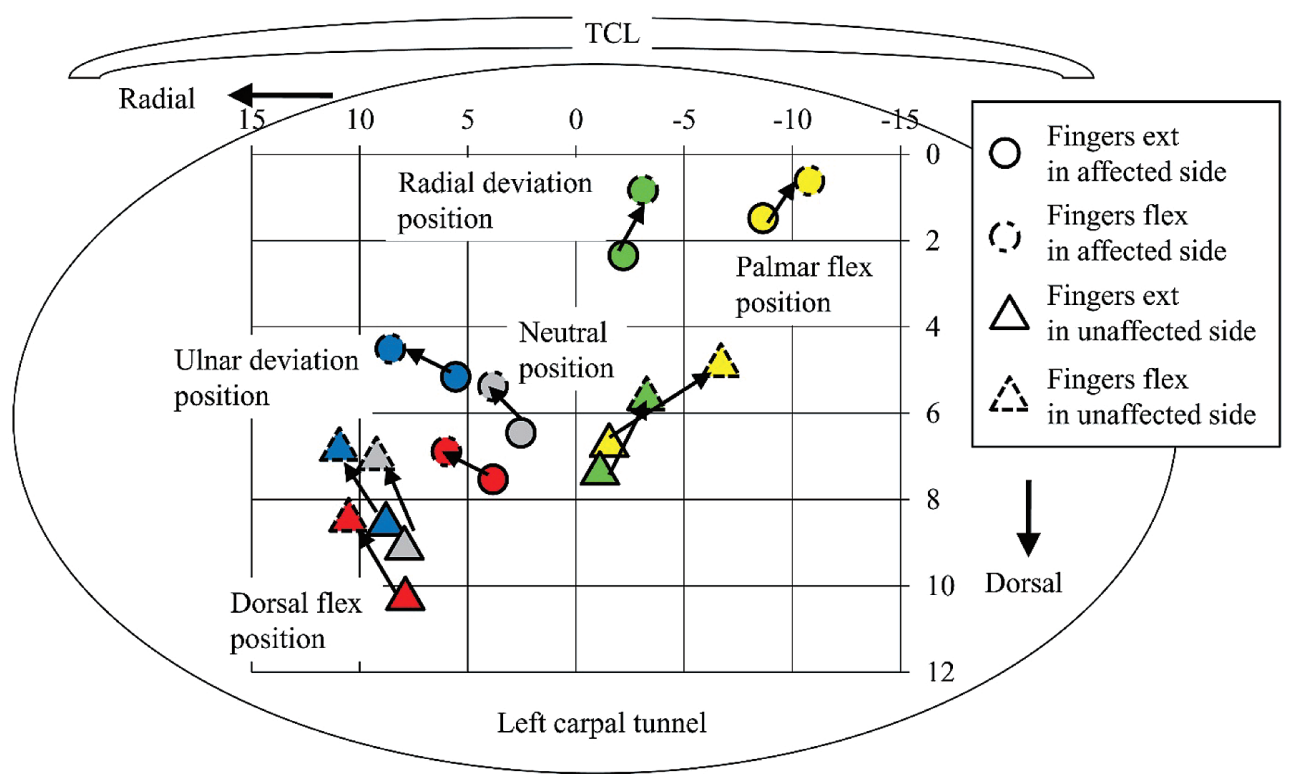

Fig. 3. Location of the median nerve during wrist and finger motion within the carpal tunnel (Average value). Gray: Wrist neutral position. Red: Wrist Dorsal flexion position. Yellow: Wrist palmar flexion position. Green: Wrist radial deviation position. Blue: Wrist ulnar deviation position.

TCL, the transverse carpal ligament. 
would be readily compressed by the flexor tendons, especially at the wrist palmar flexion position in the affected side.

In this study, the detailed transverse displacement and direction of the median nerve as a coordinate within the carpal tunnel were examined quantitatively during wrist and finger motion using ultrasound in both the affected side and the unaffected side in CTS patients (Fig. 3). This study demonstrated that there were significant differences in the median nerve displacement and the direction within the carpal tunnel between in the affected side and in the unaffected side. To better understand the detailed nerve kinematics in CTS, we compared the nerve movement in the affected side with the unaffected side of the same individual in CTS patients. In consequence, we found that at all wrist positions during finger motion, the median nerve moved significantly more ulnopalmarly in the affected side than in the unaffected side. From this research, we showed that there is a possibility that such a pattern of median nerve displacement has the potential to distinguish affected from unaffected individuals. And, this ultrasound information can be useful in the evaluation and understanding of the nerve kinematics within the carpal tunnel, and in further understanding of the pathogenesis of CTS. Additionally, it could help to improve accurate diagnosis and treatment for CTS when detecting patients. Future studies will be directed toward evaluating the median nerve movement during wrist and finger motion in CTS patients compared between before and after carpal tunnel release.

The present study had several limitations. First, we examined a small size of the participants. A larger size of the group comprising CTS patients differing in age, gender and ethnicity, may provide more definitive conclusions to our findings with regard to the median nerve displacement and CTS prevalence. It also could explain variations in detail and improve the precision in measurement of the nerve movement. Second, a weakness of this study was the reproducibility of the ultrasound measurements and analysis. All measurements were performed by a single examiner. Therefore, we could not assess interobserver reliability. Ultrasound measurements are known to be examiner dependent, especially concerning transducer placement. In the current study, a custom-made transducer fixing device was secured at the patient's hand and a table to hold the transducer stable during wrist or finger motion. Having the transducer fixed in position may help to minimize examiner dependency. Finally, the severity of CTS was not evaluated. Increasing group size may enable subgroup analysis to further investigate the relation between clinical severity and the nerve movement within the carpal tunnel.

\section{Conflict of Interest}

The authors declare no conflict of interest.

\section{References}

Ettema, A.M., Amadio, P.C., Zhao, C., Wold, L.E. \& An, K.N. (2004) A histological and immunohistochemical study of the subsynovial connective tissue in idiopathic carpal tunnel syndrome. J. Bone Joint Surg. Am., 86, 1458-1466.

Ettema, A.M., Zhao, C., Amadio, P.C., O'Byrne, M.M. \& An, K.N. (2007) Gliding characteristics of flexor tendon and tenosynovium in carpal tunnel syndrome: a pilot study. Clin. Anat., 20, 292-299.

Erel, E., Dilley, A., Greening, J., Morris, V., Cohen, B. \& Lynn, B. (2003) Longitudinal sliding of the median nerve in patients with carpal tunnel syndrome. J. Hand Surg. Br., 28, 439-443.

Liong, K., Lahiri, A., Lee, S., Chia, D., Biswas, A. \& Lee, H.P. (2014) Predominant patterns of median nerve displacement and deformation during individual finger motion in early carpal tunnel syndrome. Ultrasound Med. Biol., 40, 18101818.

Lluch, A.L. (1992) Thickening of the synovium of the digital flexor tendons: cause or consequence of the carpal tunnel syndrome? J. Hand Surg. Br., 17, 209-212.

Nakamichi, K. \& Tachibana, S. (1992) Transverse sliding of the median nerve beneath the flexor retinaculum. J. Hand Surg. Br., 17, 213-216.

Nakamichi, K. \& Tachibana, S. (1995) Restricted motion of the median nerve in carpal tunnel syndrome. J. Hand Surg. Br., 20, 460-464.

Nanno, M., Sawaizumi, T., Kodera, N., Tomori, Y. \& Takai, S. (2015) Transverse ultrasound assessment of the displacement of the median nerve in the carpal tunnel during wrist and finger motion in healthy volunteers. J. Nippon Med. Sch., [in press].

Tetro, A.M., Evanoff, B.A., Hollstien, S.B. \& Gelberman, R.H. (1998) A new provocative test for carpal tunnel syndrome: assessment of wrist flexion and nerve compression. J. Bone Joint Surg. Br., 80, 493-498.

Ugbolue, U.C., Hsu, W.H., Goitz, R.J. \& Li, Z.M. (2005) Tendon and nerve displacement at the wrist during finger movements. Clin. Biomech., 20, 50-56.

van Doesburg, M.H.M., Henderson, J., Mink van der Molen, A.B., An, K.N. \& Amadio, P.C. (2012) Transverse plane tendon and median nerve motion in the carpal tunnel: ultrasound comparison of carpal tunnel syndrome patients and healthy volunteers. PLoS One, 7, e37081.

van Doesburg, M.H.M., Yoshii, Y., Villarraga, H.R., Henderson, J., Cha, S.S., An, K.N. \& Amadio, P.C. (2010) Median nerve deformation and displacement in the carpal tunnel during index finger and thumb motion. J. Orthop. Res., 28, 13871390.

Vögelin, E., Mészàros, T., Schöni, F. \& Constantinescu, M.A. (2014) Sonographic wrist measurements and detection of anatomical features in carpal tunnel syndrome. ScientificWorldJournal, 2014, 657906.

Wang, Y., Filius, A., Zhao, C., Passe, S.M., Thoreson, A.R., An, K.N. \& Amadio, P.C. (2014a) Altered median nerve deformation and transverse displacement during wrist movement in patients with carpal tunnel syndrome. Acad. Radiol., 21, $472-480$

Wang, Y., Zhao, C., Passe, S.M., Filius, A., Thoreson, A.R., An, K.N. \& Amadio, P.C. (2014b) Transverse ultrasound assessment of median nerve deformation and displacement in the human carpal tunnel during wrist movements. Ultrasound Med. Biol., 40, 53-61.

Yoshii, Y., Ishii, T., Tung, W.L., Sakai, S. \& Amadio, P.C. (2013) Median nerve deformation and displacement in the carpal tunnel during finger motion. J. Orthop. Res., 31,1876-1880.

Yoshii, Y., Villarraga, H.R., Henderson, J., Zhao, C., An, K.N. \& Amadio, P.C. (2009) Ultrasound assessment of the displace- 
ment and deformation of the median nerve in the human carpal tunnel with active finger motion. J. Bone Joint Surg. Am., 91, 2922-2930.

Zhao, C., Ettema, A.M., Osamura, N., Berglund, L.J., An, K.N. \&
Amadio, P.C. (2007) Gliding characteristics between flexor tendons and surrounding tissues in the carpal tunnel: a biomechanical cadaver study. J. Orthop. Res., 25, 185-190. 